\title{
A new method of fitting $P-S-N$ curve for ultrahigh strength sucker rod
}

\author{
Yuangang $\mathrm{Xu}$ and Fanfan $\mathrm{Hao}^{\mathrm{a}}$ \\ Xi'an Shiyou University, Xi'an, Shaanxi, 710065, China
}

\begin{abstract}
It is commonly believed that the fatigue life and cyclic stress is logarithm linear dependence when fitting $P-S-N$ curve, but data of fatigue tests indicate that the linear correlation isn't evident for ultrahigh strength sucker rod. It is necessary to present a new fitting method. The scatter diagram of $\lg S_{P}$ and $\lg N_{P}$ is fitted by several nonlinear models in SPSS, and the model $\mathrm{S}$ has the highest goodness of fitting. Finally modified equations of $P-S-N$ curve for ultrahigh strength sucker rod are obtained.
\end{abstract}

Keywords: $P-S-N$ curve; ultrahigh strength sucker rod; correlation coefficient; nonlinear fitting.

\section{Introduction}

$P$-S $N$ curve is relation curve between fatigue life and cyclic stress processed by fatigue tests in the survival rate at a given value [1]. Under adequate alternating stress, the cross section of sucker rod gradually is weakened, and sucker rod will have fatigue failures when stress reaches a certain limit. Additionally the statistical data of oil fields suggests that about $70 \%$ failures of sucker rod are due to fatigue damage, so the $P-S$-Ncurve has practical significance.

Previous $P$ - $S$-Ncurve of sucker rod is on the basis of assumptions:

1) At an equivalent stress level fatigue life follows the normal distribution.

2) At a given survival rate, the relation of cyclic stress $S_{P}$ and fatigue life $N_{P}$ follows this equation,

$$
N_{P} S_{P}{ }^{\mathrm{m}}=C
$$

where $m$ and $C$ are constant, and $\lg S_{P}$ and $\lg N_{P}$ is linear dependent.

Based on these assumptions, $P$ - $S$ - $N$ curve of sucker rod is fitted by methods such as Least Square, Weighted Least Square, Maximum Likelihood [1-3], etc. However according to a great deal of fatigue tests of metal materials, $\lg S_{P}$ and $\lg N_{P}$ is not linear dependent for $P-S$ - $N$ curve in medium or long life region [4].

Because of high fatigue strength, ultrahigh strength sucker rod doesn't meet assumptions and it's necessary to correct the method of fitting $P-S-N$ curve.

\footnotetext{
${ }^{a}$ Corresponding author : Fan_FanHao@126.com
} 


\section{Analysis of data of fatigue tests}

Using PLG-300 fatigue-testing machine, data of fatigue life under different stress level, shown in Table 1, are acquired for ultrahigh strength sucker rod.

Table 1. Data of fatigue tests.

\begin{tabular}{|c|c|c|}
\hline Grade & Stress level (MPa) & Fatigue life (time) \\
\hline \multirow{4}{*}{ HY } & 500 & $465674,472523,582672,416596,586241$ \\
\cline { 2 - 3 } & 540 & $339481,436807,677751,685322,292733$ \\
\cline { 2 - 3 } & 600 & $250950,314635,347449,281548,491519$ \\
\hline \multirow{3}{*}{ HL } & 500 & $342935,249874,433111,413256,408352$ \\
\cline { 2 - 3 } & 540 & $274785,192247,168331,243823,240933$ \\
\cline { 2 - 3 } & 600 & $197732,285623,294802,320136,302025$ \\
\hline
\end{tabular}

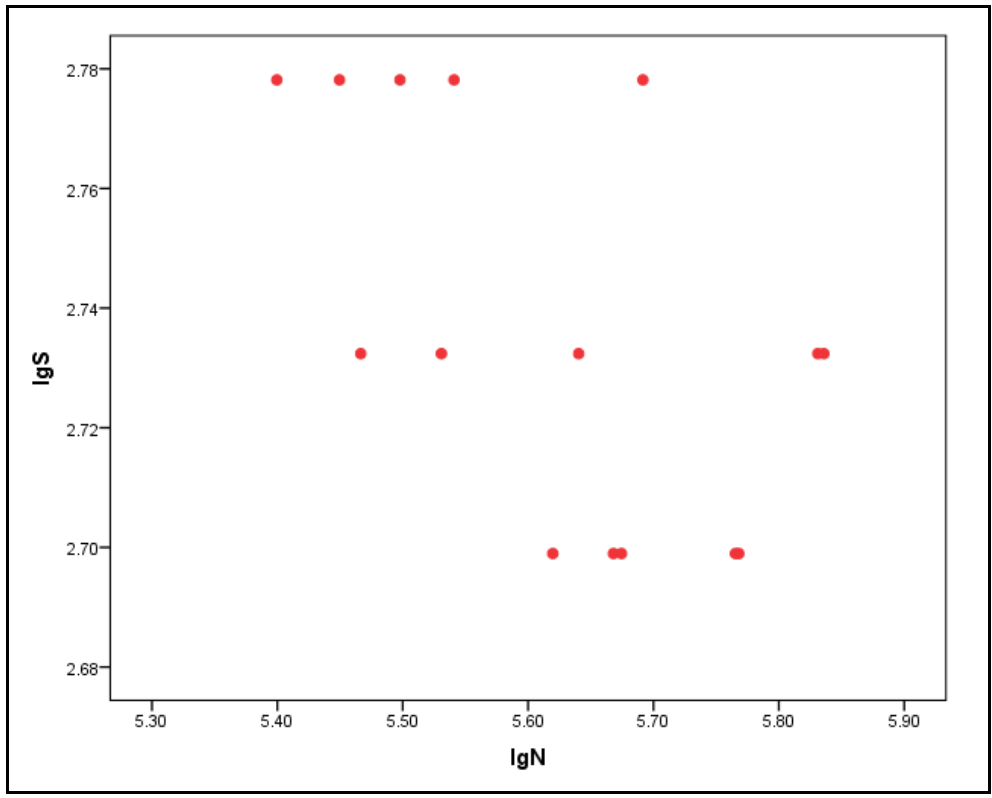

Figure 1. Scatter diagram of $\lg S_{P}$ and $\lg N_{P}$ for grade HY sucker rod

In these scatter diagrams $\lg S_{P}$ is dependent variables and $\lg N_{P}$ is independent variables as Figure 1 and Figure 2. Correlation can't be directly got, so correlation coefficient is introduced. 


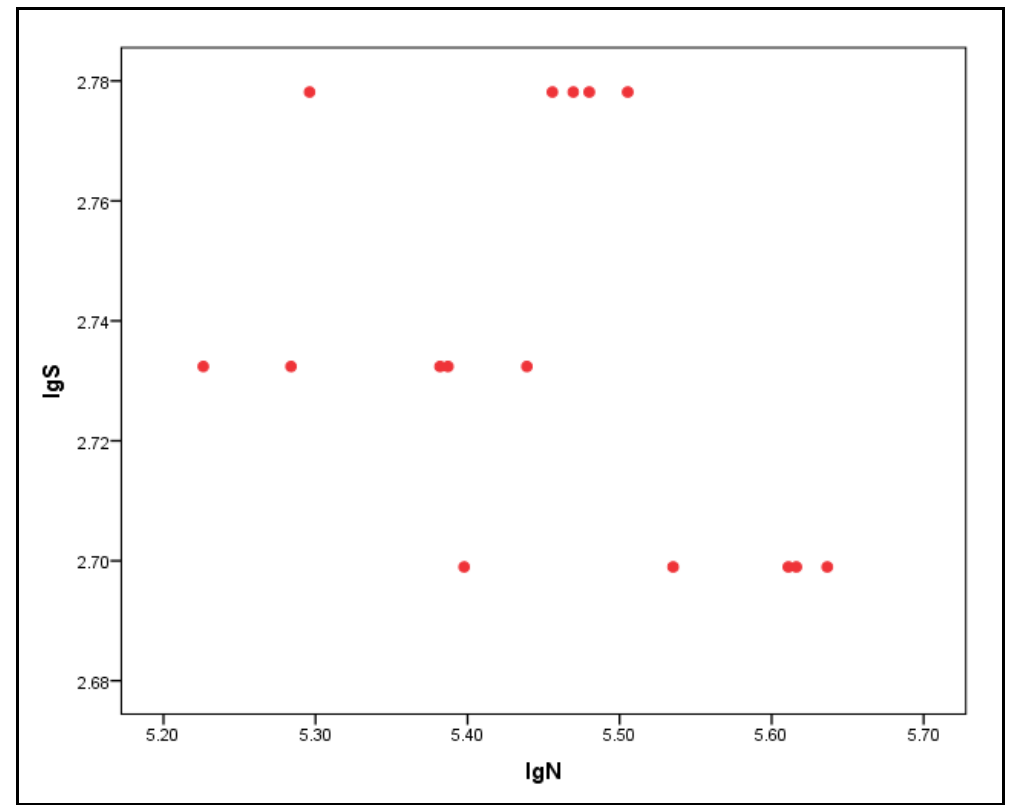

Figure 2. Scatter diagram of $\operatorname{lgSP}$ and $\operatorname{lgNP}$ for grade HL sucker rod

\section{Analysis of correlation}

The calculation results of K-S (Kolmogorov-Smirnov) show that at a equivalent stress level $\lg N_{P}$ follows the normal distribution when the significance level is 0.05 . Taking the case of grade HY suck rod, Figure 3 is the normal P-P plot of $\lg N_{P}$ under $500 \mathrm{MPa}$ stress level. In Figure 3 splashes are near $45^{\circ}$ curve, that is to say $\lg N_{P}$ follows normal distribution.

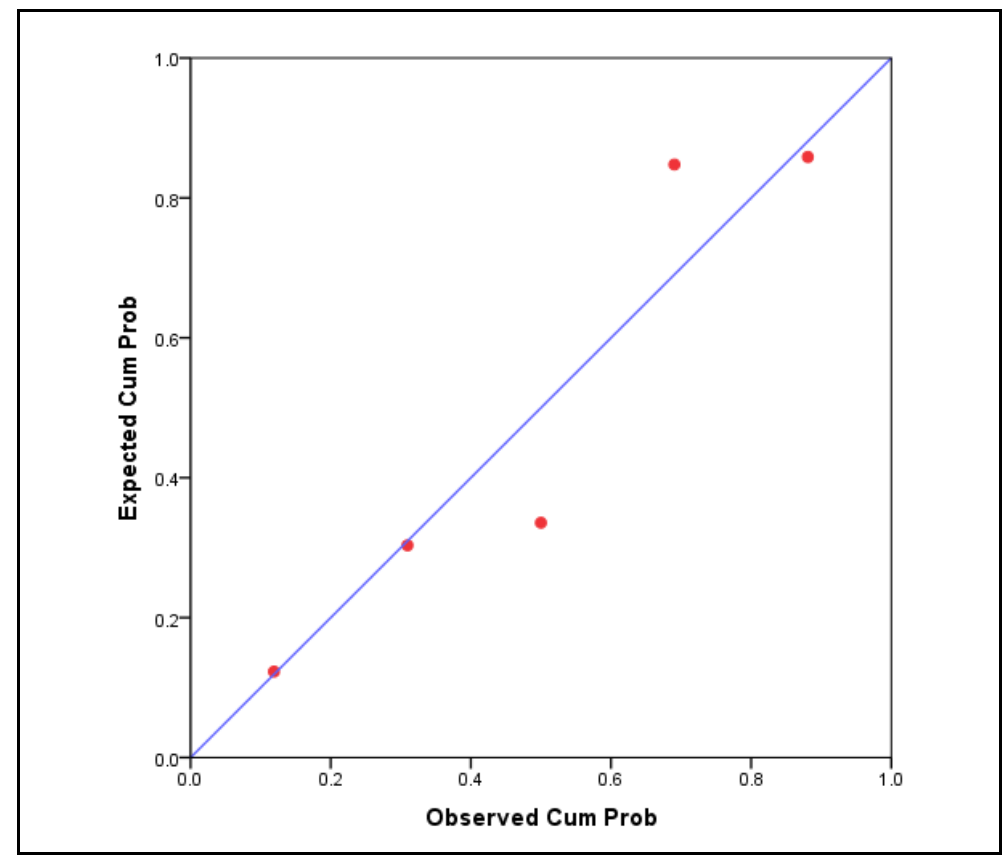

Figure 3. Normal P-P plot of $\lg N_{P}$ 
In order to evaluate the correlation of variables, correlation coefficient is introduced. Pearson simple correlation coefficient $r$ is defined as

$$
r=\frac{\sum_{i=1}^{n}\left(x_{i}-\bar{x}\right)\left(y_{i}-\bar{y}\right)}{\sqrt{\sum_{i=1}^{n}\left(x_{i}-\bar{x}\right)^{2} \sum_{i=1}^{n}\left(y_{i}-\bar{y}\right)^{2}}}
$$

Where $n$ is sample capacity, $x_{i}$ is dependent variables, $y_{i}$ is independent variables. If $r=0$, there is no linear correlation. And the nearer an absolute value of $r$ to 1 , there is more obvious linear correlation.

We propose an assumption $\mathrm{H}_{0}$ : there is no obvious linear correlation, or there is zero correlation.

Test statistics $t$ is defined as

$$
\mathrm{t}=\frac{r \sqrt{n-2}}{\sqrt{1-r^{2}}}
$$

$t$ follows the t-distribution with $n-2$ degrees of freedom.

SPSS (Statistical Product and Service Solutions) software calculate Pearson simple correlation coefficient $r$, test statistics $t$ and corresponding probability P-shown in Table 3.

Table 2. Results of calculations by SPSS.

\begin{tabular}{|c|c|c|c|}
\hline Grade & $\boldsymbol{r}$ & $\boldsymbol{t}$ & P- \\
\hline HY & -0.567 & 0.027 & \multirow{2}{*}{0.05} \\
\cline { 1 - 3 } HL & -0.347 & 0.205 & \\
\hline
\end{tabular}

For HY sucker rod, $r=-0.567$ and P- less than 0.05 , that is to say the assumption is valid, that is to say the assumption is invalid, so there is no obvious linear relationship. For HL sucker rod, $r=-0.347$ and $\mathrm{P}$ - more than 0.05 , so there is no linear relationship.

So the method of linear fitting is not suitable for fitting $P-S-N$ curve of ultrahigh strength sucker rod, and a new method of nonlinear fitting should be used.

\section{Nonlinear fitting of $P-S-N$ curve}

Proper fitting of these experimental data is completed with some nonlinear models of SPSS software, shown in Table 3, and the reliability is $95 \%$.

$R^{2}$ processed by SPSS is used to estimate the goodness of fitting with different nonlinear models. The physical significance of $R^{2}$ is correlation coefficient squared between independent variables and dependent variables, and the higher value of $R^{2}$ indicates a higher adoption of fitting models. It is assumed that the fitting model of grade HY and HL sucker rod is the same, and the results are shown in Table 4. is $95 \%$.

Finally equations of $P-S-N$ curve for ultrahigh strength sucker rod are obtained when survival rate 
Table 3. Equations of nonlinear models in SPSS.

\begin{tabular}{|c|c|}
\hline Model & Equation \\
\hline Compound & $\mathrm{y}=\beta_{0} \beta_{1}{ }^{x}$ \\
\hline Growth & $\mathrm{y}=e^{\beta_{0}+\beta_{1} x}$ \\
\hline Logarithmic & $y=\beta_{0}+\beta_{1} \ln (x)$ \\
\hline $\mathrm{S}$ & $\mathrm{y}=e^{\beta_{0}+\beta_{1} / x}$ \\
\hline Exponential & $\mathrm{y}=\beta_{0} e^{\beta_{1} x}$ \\
\hline Power & $\mathrm{y}=\beta_{0}\left(x^{\beta_{1}}\right)$ \\
\hline Logistic & $y=\frac{1}{1 / \mu+\beta_{0} \beta_{1} x}$ \\
\hline
\end{tabular}

Table 4. Results of nonlinear fitting.

\begin{tabular}{|c|c|c|c|c|}
\hline Grade & Model & $\beta_{0}$ & $\beta_{1}$ & Equations of fitting curve \\
\cline { 1 - 4 } & \multirow{2}{*}{$\mathrm{HY}$} & 0.725 & 1.584 & $\lg S=e^{0.725+1.584 / \lg N}$ \\
\cline { 1 - 3 } & $\mathrm{S}$ & 0.823 & 0.999 & $\lg \mathrm{S}=e^{0.823+0.999 / \lg N}$ \\
\hline
\end{tabular}

For grade HY sucker rod,

$$
\lg S=e^{0.725+1.584 / \lg N}
$$

For grade HL sucker rod,

$$
\lg \mathrm{S}=e^{0.823+0.999 / \lg N}
$$

And figures of $P-S-N$ curve for ultrahigh strength sucker rod are shown in Figure 4 and Figure 5.

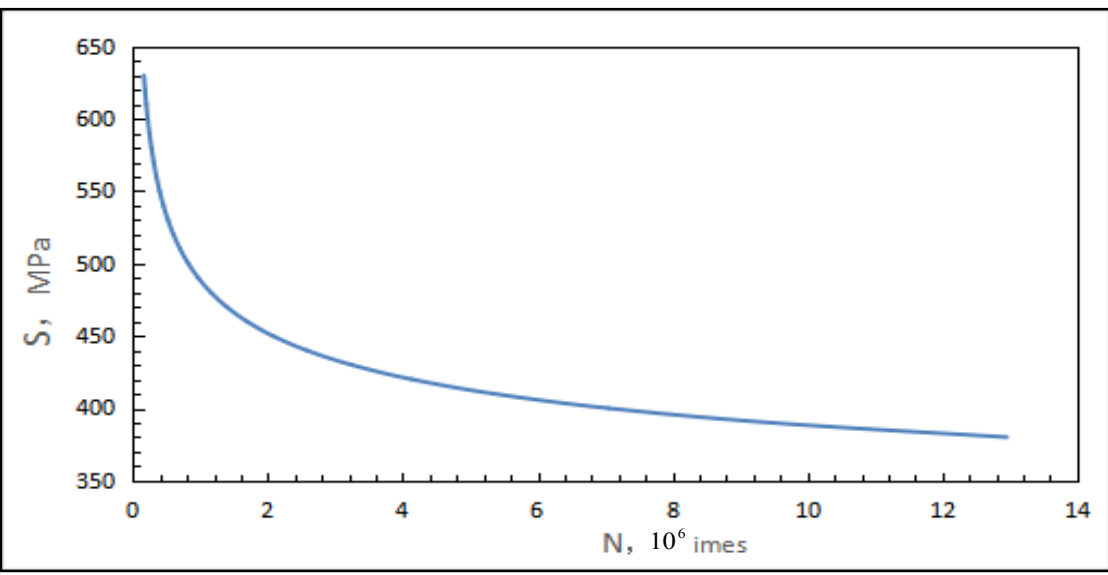

Figure 4. $P-S-N$ curve of grade HY sucker rod. 


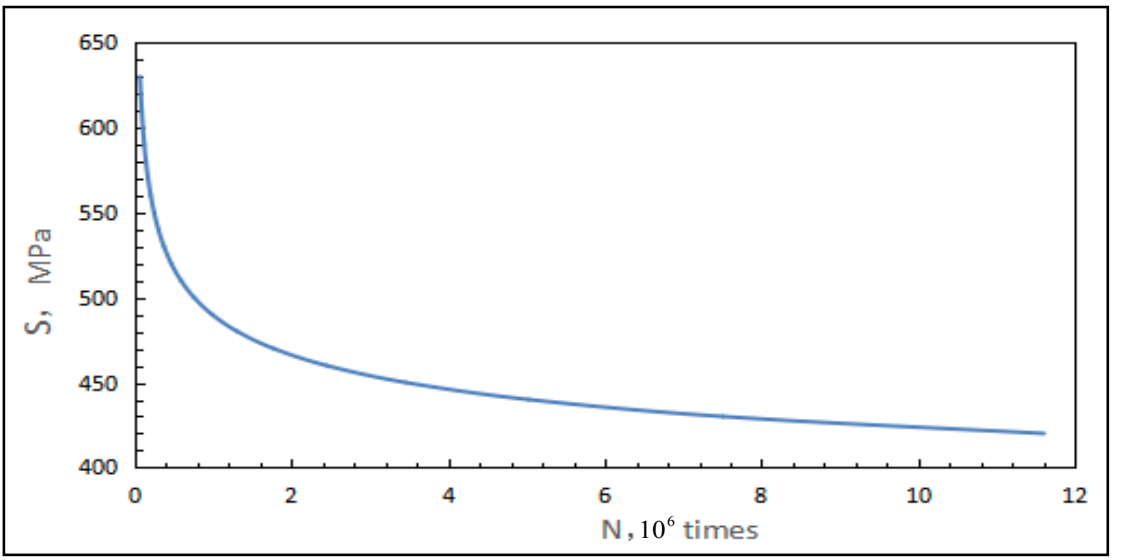

Figure 5. P-S-N curve of grade HL sucker rod.

\section{Summary}

According to analysis of correlation coefficient, the relation of $\lg S_{P}$ and $\lg N_{P}$ is not simple $\operatorname{linear}$ correlation. A nonlinear method is used to fit data of fatigue tests for ultrahigh strength sucker rod, and equations of $S-P$ - $N$ curve are got. Equations can provide theoretical references for scientific use of ultrahigh strength sucker rod to avoid fatigue damage.

\section{Acknowledgments}

This work was financially supported by Fund Project of Excellent Master Degree Thesis Cultivation of Xi'an Shi You University (2015yp140104) and Innovation Fund Project of Xi'an Shiyou University Graduate Students (2015cx140104).

\section{References}

1. Liyang Xie, Jianzhong Liu. Principle of Samples Polymerization and Method of $P-S-N$ Curve Fitting. Journal of Mechanical Engineering. 49, 96-103 (2013)

2. Kailai Song. Experimental Research of $P-S-N$ Curves for Sucker Rod with Super High Strength. Oil Field Equipment. 32, 27-29 (2003)

3. Peng Gao, Bo Zhang, Peng He. Fatigue Life Mode of Grade H Sucker Rod. China Petroleum and Chemical Standard and Quality. 187-188 (2014)

4. Huimin $\mathrm{Fu}$, Zhentong Gao, Meixun Liang. A Method for Fitting $P-S-N$ Curve. Acta Aeronautica et Astronautica Sinica. 9, 399-341 (1988) 